\title{
Doppler-shift oscillations in solar spicules
}

\author{
T. V. Zaqarashvili, E. Khutsishvili, V. Kukhianidze, and G. Ramishvili
}

\author{
Georgian National Astrophysical Observatory (Abastumani Astrophysical Observatory), Kazbegi Ave. 2a, Tbilisi 0160, Georgia \\ e-mail: temury@genao.org
}

Received 18 April 2007 / Accepted 11 August 2007

\section{ABSTRACT}

\begin{abstract}
Aims. We analysed the consecutive height series of $\mathrm{H} \alpha$ spectra in solar limb spicules taken on the $53 \mathrm{~cm}$ coronagraph of Abastumani Astrophysical Observatory at the heights of $3800-8700 \mathrm{~km}$ above the photosphere. Our aim is to observe oscillatory phenomena in spicules and consequently to trace wave propagations through the chromosphere.

Methods. We use the Discrete Fourier Transform analysis of $\mathrm{H} \alpha$ Doppler shift time series constructed from the observed spectra at each height.

Results. Doppler velocities of solar limb spicules show oscillations with periods of 20-55 and 75-110 s. There is also clear evidence of 3-min oscillations at the observed heights.

Conclusions. The oscillations can be caused by wave propagations in thin magnetic flux tubes anchored in the photosphere. We suggest the granulation as a possible source of the wave excitation. Observed waves can be used as a tool for spicule seismology, and the magnetic field strength in spicules at the height of $\sim 6000 \mathrm{~km}$ above the photosphere is estimated as $12-15 \mathrm{G}$.
\end{abstract}

Key words. Sun: chromosphere - Sun: oscillations

\section{Introduction}

It is widely believed that the energy source responsible for heating the coronal plasma up to $1 \mathrm{MK}$ is located in a denser and dynamic photosphere. Chromospheric, structured magnetic fields may guide energy towards the corona and can be dissipated there, thereby heating the ambient plasma. One of most plausible mechanisms for this energy transport are the magnetohydrodynamic (MHD) waves. The waves can be generated in photospheric magnetic tubes by the buffeting of granular motions (Roberts 1979; Spruit 1981). Then they may propagate along the chromospheric magnetic field, penetrate the corona and deposit the energy into heat. Therefore observations of oscillatory motions in the chromosphere is a key test of the wave heating theory.

The most pronounced features of the chromosphere in quiet Sun regions are spicules: jet-like limb structures observed mainly in the $\mathrm{H} \alpha$ line (Beckers 1972). They are concentrated between supergranule cells and thus are probably formed in regions of intense magnetic field concentrations, although the formation mechanism is not known yet (Sterling 2000; but see Roberts 1979; and De Pontieu et al. 2004). On the other hand, spicules may arise along the magnetic tubes, which at the same time guide MHD waves from the photosphere into the corona. Therefore the wave propagation in the chromosphere may be traced through the oscillatory dynamics of spicule plasma.

Oscillations in spicules have been observed mostly with a $\sim 5$ min period (Kulidzanishvili \& Zhugzhda 1983; De Pontieu et al. 2003; Xia et al. 2005) and are probably connected with global p-modes. On the other hand, oscillations in spicules with shorter periods ( $\sim 1 \mathrm{~min}$ ) have been reported by Nikolsky \& Platova (1971) as periodic transversal displacements of spicule axes at one particular height. Recent observations of higher-frequency waves in the "green" coronal line during the August 1999 total solar eclipse (Katsiyannis et al. 2003), in the
Fe I $5434 \AA$ line by the German Vacuum Tower Telescope on Tenerife (Wunnenberg et al. 2002) and in the transition region spectral lines by TRACE (Deforest 2004; de Wijn et al. 2005), indicate the significant power at the high frequency branch of oscillations in almost the whole solar atmosphere. This stimulates more searches for short-period oscillations in spicules. These short-period waves may give significant input into chromospheric and coronal heating. Note that the excitation of shortperiod waves (10-20 s) in photospheric magnetic flux tubes has recently been proposed by Zaqarashvili \& Roberts (2002).

Kukhianidze et al. (2006, hereinafter Paper I) report periodic spatial distributions of Doppler velocities with height through spectroscopic analysis of $\mathrm{H} \alpha$ height series in solar limb spicules (3800-8700 km above the photosphere). They find that nearly $20 \%$ of the measured height series show a periodic spatial behaviour with $\sim 3500 \mathrm{~km}$. This spatial periodicity in Doppler velocities was explained as a signature of kinkwave propagation in spicules. Wave periods were estimated as 35-70 s based on the expected kink speed in the chromosphere $\left(50-100 \mathrm{~km} \mathrm{~s}^{-1}\right)$. They suggest that the observed wave length is shorter $(\sim 800-1000 \mathrm{~km})$ at the photospheric level due to the decrease in kink speed. The estimated wave length at the photospheric level is comparable to the spatial dimensions of granular cells, so the granulation was suggested as a possible source of the wave excitation.

Observations of the waves may lead to a solution for coronal heating problem. Therefore, to seek oscillatory motions in the chromosphere, we performed a complete analysis of $\mathrm{H} \alpha$ series, the preliminary results of which were presented in the Paper I. Here we study the temporal dynamics of consecutive $\mathrm{H} \alpha$ spectra with a time interval of $\sim 7-8 \mathrm{~s}$ between consecutive measurements at fixed heights, which cover almost a whole life time of spicules (7-15 $\mathrm{min})$. We show spectroscopic evidence of wave propagation in the chromosphere in terms of Doppler velocity oscillations in time at specific heights of solar limb spicules. 

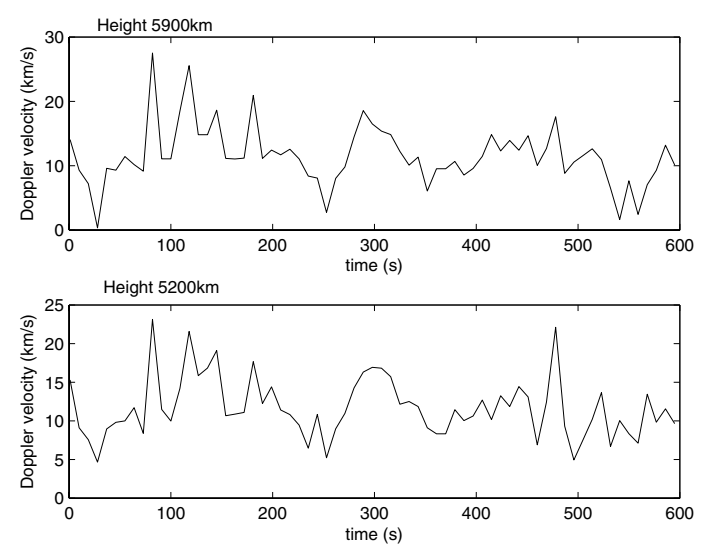

Fig. 1. Doppler velocity time series at the third $(\sim 5200 \mathrm{~km})$ and fourth $(\sim 5900 \mathrm{~km})$ heights in the spicule I. The time interval between consecutive measurements is $\sim 8 \mathrm{~s}$.

\section{Observation and data analysis}

Observations were carried out on the big $(53 \mathrm{~cm})$ coronagraph of Abastumani Astrophysical Observatory (instrumental spectral resolution and dispersion in $\mathrm{H} \alpha$ are $0.04 \AA$ and $1 \AA / \mathrm{mm}$ correspondingly) on September 26, 1981 at the solar limb as height series beginning at $3800 \mathrm{~km}$ height from the photosphere and upwards (for details, see Khutsishvili 1986). The chromospheric $\mathrm{H} \alpha$ line was used to observe solar limb spicules at 8 different heights. The distance between neighbouring heights was 1 " (which was the spatial resolution of observations), so that the distance $\sim 3800-8700 \mathrm{~km}$ above the photosphere was covered. The exposure time was $0.4 \mathrm{~s}$ at the four lower heights and $0.8 \mathrm{~s}$ at higher ones. The total duration of each height series was $7 \mathrm{~s}$. The consecutive height series begins immediately. Therefore continuous time series of $\mathrm{H} \alpha$ spectra with interval of $\sim 7-8 \mathrm{~s}$ between consecutive measurements at each height can be constructed. These time series cover almost a whole lifetime (from 7 to $15 \mathrm{~min}$ ) of several spicules.

Each $\mathrm{H} \alpha$ line profile from the time series was fitted to a Gaussian. Then temporal variations of the Gaussian centre with respect to the photospheric reference line (4371 $\AA$ ) were studied at each heights for four different spicules. We calculated the Doppler shifts, consequently Doppler velocities, with 7-8 s interval at each height in all spicules. Figure 1 shows the Doppler velocity time series at third $(\sim 5200 \mathrm{~km})$ and fourth $(\sim 5900 \mathrm{~km})$ heights in one of the spicules. Then the spectral analysis of the time series at all heights was carried out with the Discrete Fourier Transform (DFT). DFT enables oscillation periods to be revealed with 20-250 s; the shorter periods are restricted due to an interval between consecutive measurements, while the longer periods are restricted due to the lifetimes of spicules.

\section{Results}

In this section, we present the results of DFT in four different spicules separately. Best fits of $\mathrm{H} \alpha$ line profiles to a Gaussian were found at third $(\sim 5200 \mathrm{~km})$ and fourth $(\sim 5900 \mathrm{~km})$ heights. The fits were relatively poor at lower and higher heights. Therefore calculated Doppler shifts are more confident at the third and fourth heights. For this reason, we first show the results of DFT for these heights, then turn to general oscillatory phenomena at all heights.
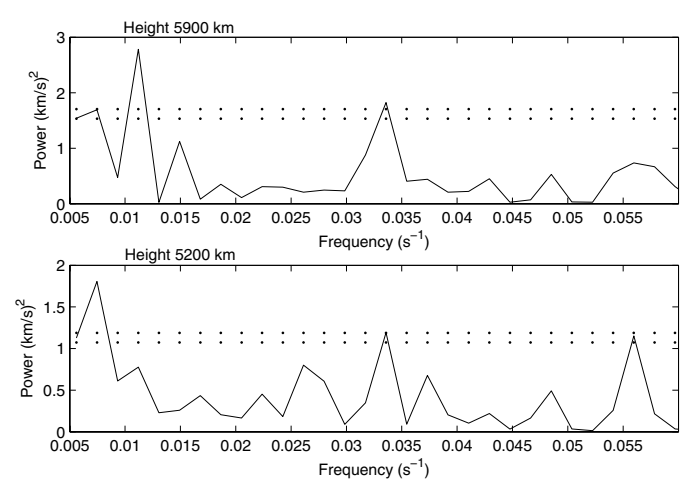

Fig. 2. Power spectra of Doppler velocity oscillations in the spicule I at the heights of $5200 \mathrm{~km}$ (lower panel) and 5900 (upper panel). The dotted lines in both plots show $95.5 \%$ and $98 \%$ confidence levels.
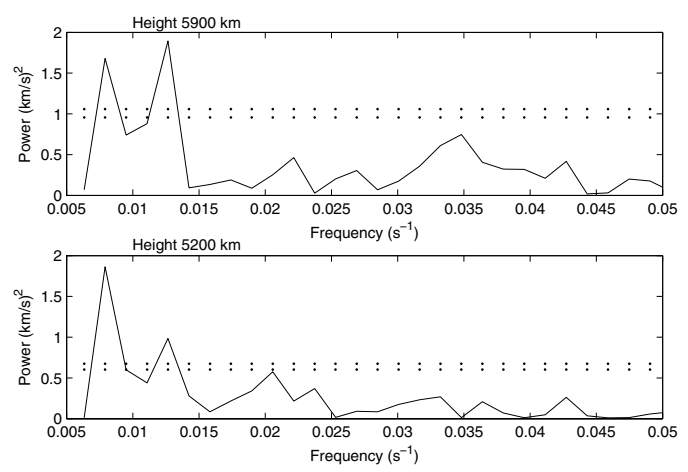

Fig. 3. Power spectra of Doppler velocity oscillations in the spicule II at the heights of $5200 \mathrm{~km}$ (lower panel) and $5900 \mathrm{~km}$ (upper panel). The dotted lines show $95.5 \%$ and $98 \%$ confidence levels.

\subsection{Spicule I}

Figure 2 shows the power spectra of Doppler velocity oscillations in the spicule I at the heights of $5200 \mathrm{~km}$ (below) and $5900 \mathrm{~km}$ (up).

The most pronounced periods at the height of $5200 \mathrm{~km}$ are 180, 30, and $17 \mathrm{~s}$ (Fig. 2, lower panel). However, the $17 \mathrm{~s}$ period is probably suspicious as it is closer to the time interval between consecutive measurements $(\sim 7-8 \mathrm{~s})$, so the oscillations of Doppler velocity with the periods of 180 and 30 s are more confident at this height. The upper panel shows the power spectrum at the height of $5900 \mathrm{~km}$. Here the most pronounced periods are 180,90 , and $30 \mathrm{~s}$, so the spicule oscillates with the periods of 30 and $180 \mathrm{~s}$ at both heights. The oscillation with the period of $90 \mathrm{~s}$ is also seen but preferably at the higher height (but note the small peak at the lower height as well).

\subsection{Spicule II}

Figure 3 shows the power spectra of Doppler velocity oscillations in the spicule II at the heights of $5200 \mathrm{~km}$ (lower panel) and $5900 \mathrm{~km}$ (upper panel). We see two clear oscillation periods of 120 and $80 \mathrm{~s}$ at both heights. Both periods are above the $98 \%$ confidence level. There is some evidence of a $\sim 50 \mathrm{~s}$ period at the height of $5200 \mathrm{~km}$, but just below of the $95.5 \%$ confidence level.

\subsection{Spicule III}

Figure 4 shows the power spectra of Doppler velocity oscillations in the spicule III at the heights of $5200 \mathrm{~km}$ (lower panel) 

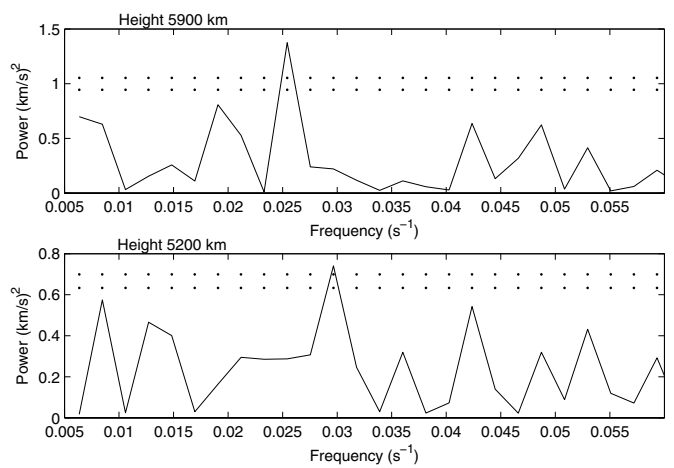

Fig. 4. Power spectra of Doppler velocity oscillations in the spicule III at the heights of $5200 \mathrm{~km}$ (lower panel) and $5900 \mathrm{~km}$ (upper panel). The dotted lines show $95.5 \%$ and $98 \%$ confidence levels.
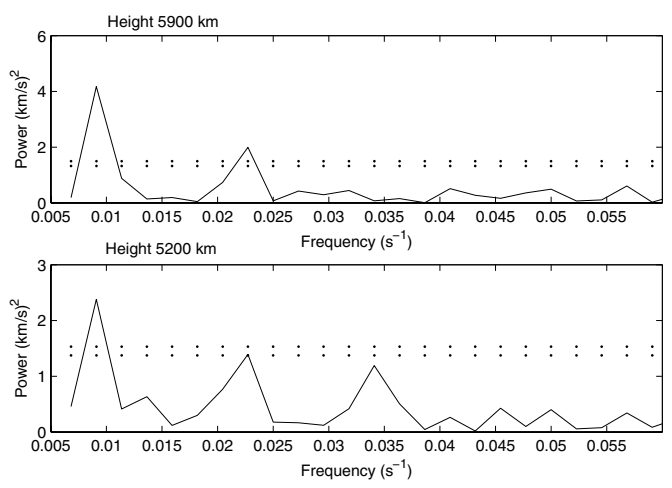

Fig. 5. Power spectra of Doppler velocity oscillations in the spicule IV at the heights of $5200 \mathrm{~km}$ (lower panel) and $5900 \mathrm{~km}$ (upper panel). The dotted lines show $95.5 \%$ and $98 \%$ confidence levels.

and $5900 \mathrm{~km}$ (upper panel). The spicule shows the oscillations with $37 \mathrm{~s}$ period at the height of $5200 \mathrm{~km}$ and with a $35 \mathrm{~s}$ period at the height of $5900 \mathrm{~km}$, hence, it oscillates with the period of $\sim 35 \mathrm{~s}$ at both heights.

\subsection{Spicule IV}

Figure 5 shows the power spectra of Doppler velocity oscillations in the spicule IV at the heights of $5200 \mathrm{~km}$ (lower panel) and $5900 \mathrm{~km}$ (upper panel). Clear oscillation periods in this spicule at both heights are $\sim 110$ and $\sim 40 \mathrm{~s}$. There is evidence of oscillations with $30 \mathrm{~s}$, but just below of the $95.5 \%$ confidence level.

\subsection{All oscillatory periods above the $95.5 \%$ confidence level}

Now we present the results of DFT for all 32 time series; i.e. at 8 different heights in 4 different spicules. Figure 6 shows a histogram of all the oscillation periods above the $95.5 \%$ confidence level. The histogram reveals interesting properties of the oscillatory periods in spicules. Almost half of the oscillatory periods are located in the range of 18-55 s, which was suggested by Kukhianidze et al. in Paper I. Another interesting range of oscillatory periods is $75-110 \mathrm{~s}$, with a clear peak at the period of $90 \mathrm{~s}$. Note the interesting peak at the $178 \mathrm{~s}$ period as well, which is clear evidence of well-known 3 min oscillations.

To show the spatial locations of oscillations, we plot a Fourier power (expressed in confidence levels) as a function of frequency and heights for spicules II (Fig. 7, upper panel) and IV (lower panel). There is clear evidence of persisting

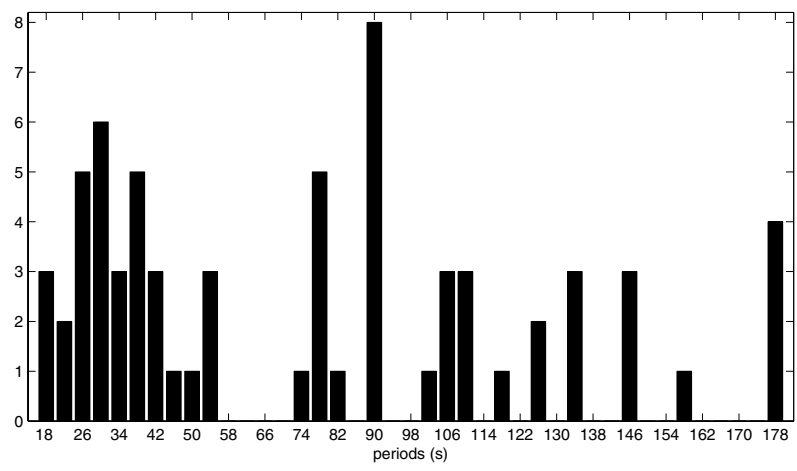

Fig. 6. Histogram of all oscillation periods that are above $95.5 \%$ confidence level. The horizontal axis shows the oscillation periods in seconds, while the vertical axis shows the number of corresponding periods.
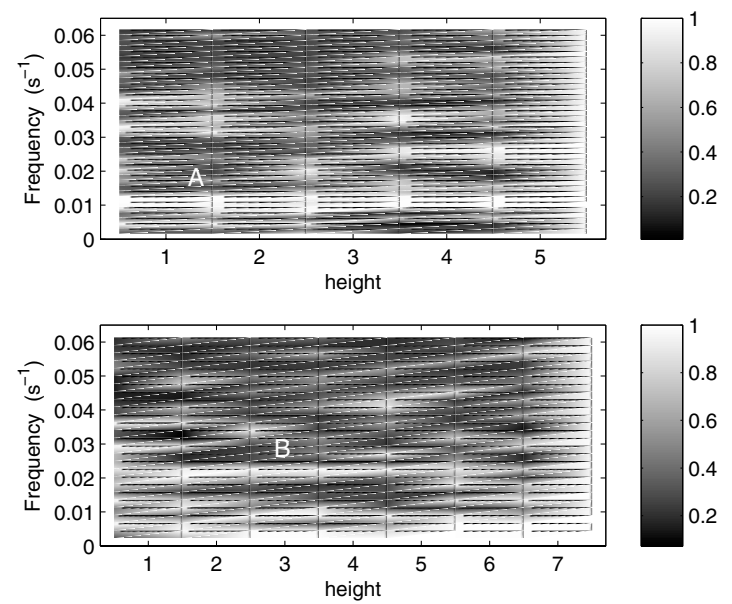

Fig. 7. Fourier power expressed in confidence levels as a function of frequency and heights for the spicules II (upper panel) and IV (lower panel). Light points correspond to higher power, and dark points correspond to lower one. The label 1 on the power scale (right plots) corresponds to the $100 \%$ confidence level. In the upper plot, we see a long light feature located just above the frequency $0.01 \mathrm{~s}^{-1}$ (feature A). In the lower plot, there is another long feature (B) located above the frequency $0.02 \mathrm{~s}^{-1}$.

oscillations along the full length of both spicules. The plot of the spicule II shows the long white feature (feature A) located just above the frequency $0.01 \mathrm{~s}^{-1}$. This is the oscillation with the period of $\sim 80 \mathrm{~s}$ found at third and fourth heights (see Sect. 3.2). The oscillation persists along the spicule and is a signature of either a standing or a propagating wave pattern. The most pronounced feature (feature B) in the plot of the spicule IV is a long light trend located just above the frequency of $0.02 \mathrm{~s}^{-1}$ and persisting along almost the whole spicule. This is the oscillation with a period of $44 \mathrm{~s}$ (the same period was found at third and fourth heights; see Sect. 3.4). Thus there is the wave pattern with a $\sim 40-45$ s period in the spicule IV. However, it must be mentioned again that only the oscillations at third and fourth heights have a high confidence due to the good fit of line profile to a Gaussian.

\section{Discussion}

Time series of $\mathrm{H} \alpha$ spectra in solar limb spicules show clear evidence of Doppler shift oscillations, which probably are caused by oscillations in the line-of-sight velocity. Spicules have almost 
a vertical direction at the solar limb, therefore the velocity is probably transversal to spicule axes. However, longitudinal oscillations also cannot be ruled out if spicule axes are tilted away from the vertical. It is clear that the oscillations in Doppler velocity can be caused by wave propagations in spicules.

\subsection{Wave propagation in spicules}

Photospheric granulation is often suggested as a source of wave excitations in anchored thin magnetic tubes (Roberts 1979; Spruit 1981; Hollweg 1981; Hasan \& Kalkofen 1999; Mishonov et al. 2007). The waves may propagate along the tubes towards the corona and carry the energy and momentum. The tubes may guide spicule material at the same time. Therefore the wave propagation in the chromosphere may be traced through spicule dynamics. Magnetic tubes may guide three different types of waves: kink, sausage, and torsional Alfvén waves. Some of these waves may cause the observed Doppler-shift oscillations. Torsional Alfvén waves in thin tubes may lead to periodic non-thermal broadenings of spectral lines, but not to Dopplershift oscillations (Zaqarashvili 2003; Zaqarashvili \& Murawski 2007). Sausage waves cause oscillations mainly in a line intensity due to density variations. However, the longitudinal velocity field of sausage waves may lead to the Doppler shift variations if tube axes are significantly tilted away from the vertical. But the main contributor to Doppler-shift oscillations at the solar limb probably are kink waves, which oscillate transversally to the tube axis. We argue that the back-and-forth transversal motions of vertical tube axis at the solar limb due to the propagation of kink waves is the most plausible source for observed Dopplershift oscillations in spicules (Kukhianidze et al. 2005).

Photospheric granulations may excite waves in anchored magnetic tubes with two different time scales. The first time scale can be similar to the lifetimes of granular cells, which gives wave periods of $\sim 5-10 \mathrm{~min}$. The second time scale can be related to the spatial scales of granular cells; i.e. waves are excited with wave lengths corresponding to cell spatial dimensions $(\sim 200-1000 \mathrm{~km})$. Then these waves may have periods of 20-100 s corresponding to typical photospheric wave speed $\sim 10 \mathrm{~km} \mathrm{~s}^{-1}$. Both sound and Alfvén speeds probably have similar values in the photosphere. Figure 6 shows that more than $2 / 3$ of oscillation periods are located in the range of 20-100 s, which clearly indicates the granular origin of the waves. It is seen that the periods are split into two different ranges: 20-55 and $75-110 \mathrm{~s}$.

The reason for this phenomenon is not clear. There are two potential candidates for this splitting: either an unknown scaling of granular cells or fast and slow modes in thin tubes. The first case implies the scaling of granular cells into two different ranges of 200-500 and 800-1100 km, which is not known (but would be interesting to look into this problem in the future). The second case implies the propagation of fast and slow waves in spicules. There are two different characteristic speeds of wave propagation in a magnetic tube embedded in a field-free environment. These are kink and tube speeds (Edwin \& Roberts 1983)

$c_{\mathrm{k}}=c_{\mathrm{A}} \sqrt{\frac{\rho_{0}}{\rho_{0}+\rho_{\mathrm{e}}}}, \quad c_{\mathrm{T}}=\frac{c_{0} c_{\mathrm{A}}}{\sqrt{c_{\mathrm{A}}^{2}+c_{0}^{2}}}$,

where

$c_{\mathrm{A}}=\frac{B_{0}}{\sqrt{4 \pi \rho_{0}}}, \quad c_{0}=\sqrt{\frac{\gamma p_{0}}{\rho_{0}}}$ are the Alfvén and sound speeds inside the tube, respectively. Here $p_{0}, \rho_{0}$ and $B_{0}$ are the pressure, the density, and the magnetic field inside the tube, $\rho_{\mathrm{e}}$ the density outside the tube and $\gamma$ the ratio of specific heats.

Fast waves propagate with a phase speed close to the kink speed, while slow waves propagate with the tube speed. The density is much higher inside the spicule than outside, therefore the kink speed is close to the Alfvén speed. If the Alfvén speed is three times faster than the sound speed in spicules at observed heights $(4000-8000 \mathrm{~km})$; i.e. $c_{\mathrm{A}}=3 c_{0}$, then $c_{\mathrm{k}} \approx 3 c_{\mathrm{T}}$ and the fast waves propagate three times faster than the slow waves. If both waves are excited with similar wave length, then the period of fast waves must be three times shorter than that of slow waves. It is intriguing that the ranges $20-55$ and $75-110$ s show a similar relation. Then the oscillations with $20-55$ s period can be caused by the propagation of fast waves, while the oscillations with 75-110 s can be due to the slow waves.

It is an interesting fact that the oscillations with 75-110 s have significant power, even more than ones with shorter period. The oscillations may be signatures of periodic vertical flows excited by the resonant buffeting of granular cells (Roberts 1979). In this interesting paper, Roberts suggested that the quasiperiodic external buffeting of granules on magnetic tubes may lead to periodic resonant vertical flows, just as squeezing of a hosepipe drives a jet of water. He took $\sim 5 \mathrm{~min}$ for the period of the external forcing corresponding to mean lifetime of granules. Consequently, resonant periodic flows must propagate with the tube speed and have the same $\sim 5$ min period. However, there are usually 3-4 granular cells in the neighbourhood of each photospheric vertical tube, which causes quasi-periodic squeezing of the tube. Then, the mean period of external forcing will be 3-4 times shorter than mean lifetime of granules. Consequently, the resonant periodic flows will have 80-100 s period, which surprisingly coincides with the observed periods. This phenomenon needs more vigorous analysis, as it may deal with spicule formation mechanism, but this is beyond scope of this paper.

Another interesting result is the clear peak at the period of $178 \mathrm{~s}$ (Fig. 6), which probably indicates oscillations with a period of $3 \mathrm{~min}$. Thus the 3 -min oscillations may penetrate up to heights of $4000-8000 \mathrm{~km}$. It is interesting to check whether 5-min oscillations are present at these heights. Unfortunately, a statistical search of this period in our data is restricted due to the lifetimes of spicules.

It is particularly important to understand whether the oscillations are due to propagating or standing wave patterns. There are only 7-8 spatial points (corresponding to each height) in our data, therefore it is not easy to infer the phase propagation. In Paper I, we presented an illustrative example of phase propagation, but it needs more vigorous treatment. The wave propagation can be revealed through the variation in Fourier phase with position (Molowny-Horas et al. 1997). Unfortunately, using the method in our data is complicated, as the oscillations have high confidence only at two heights (third and fourth ones). The oscillations at the other 6 heights are not very confident due to a poor fit of line profile to Gaussian. However, some rough estimations still can be made.

We calculated the relative Fourier phase between heights for the most pronounced features (features A and B) of Fig. 7. Figure 8 shows the relative Fourier phase as a function of heights for (a) feature A and (b) feature B. There is almost no phase difference between oscillations at different heights for feature A, which probably indicates a standing-wave like pattern with period of $\sim 80-90$ s. On the contrary, there is the significant linear 

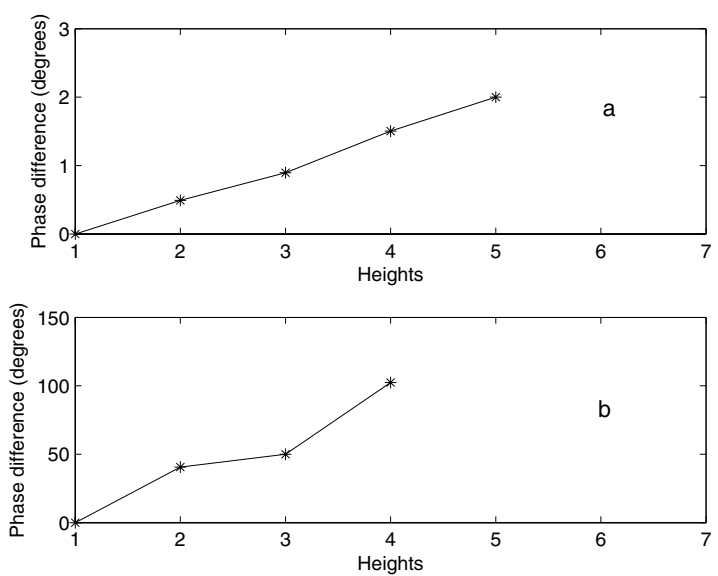

Fig. 8. Relative Fourier phase as a function of heights for a) $\sim 80 \mathrm{~s}$ period oscillation in spicule II (feature A in the upper panel of Fig. 7) and b) $44 \mathrm{~s}$ period oscillation in spicule IV (feature B in the lower panel of Fig. 7).

phase shift in the plot (b), which indicates a propagating pattern with period of $\sim 40-45 \mathrm{~s}$, so the spicule II shows the standingwave like pattern (or wave propagation almost along line of sight, which seems unlikely) and spicule IV shows the propagation pattern. The propagation speed for feature B can be roughly estimated. The wave length can be given as (Molowny-Horas et al. 1997)

$\lambda=\frac{2 \pi}{\Delta \phi} \Delta s$,

where $\Delta \phi$ and $\Delta s$ are phase difference and distance between the first and last points. Then it gives the upper limit for wave length as $\sim 6000 \mathrm{~km}$. The actual wave length will be shorter if spicule is tilted away from the vertical. For the $35^{\circ}$ tilt (Trujillo Bueno et al. 2005) the actual wave length is $4800 \mathrm{~km}$. Then the phase speed of the feature B (with period of $44 \mathrm{~s}$ ) can be estimated as

$\sim 110 \mathrm{~km} \mathrm{~s}^{-1}$,

which is near the kink speed in the chromosphere. Thus, the feature $B$ is probably due to the kink-wave propagation in the spicule IV. The origin of the feature A is controversial. The oscillations are almost in phase at 5 different heights, which indicates to a standing-wave like pattern. The pattern can be formed due to the reflection of slow tube waves at the transition to the high-temperature corona. But the phase difference analysis is not very confident in our case, so the results can be spurious. Future detailed observations are necessary to confirm this phenomenon.

\subsection{Spicule seismology}

Observed waves can be used to infer plasma parameters inside spicules. The method called coronal seismology is widely used in coronal loops (Nakariakov \& Ofman 2001) and prominences (Ballester 2006). In Paper I we reported periodical distributions of $\mathrm{H} \alpha$ Doppler velocity with height. The spatial distribution was explained in terms of kink-wave propagation from the photosphere towards the corona. The wave length was estimated as $\sim 3500 \mathrm{~km}$. Then we may calculate wave phase speed with the help of the observed oscillation period, which enables us to estimate a magnetic field strength in spicules. Singh \& Dwivedi (2007) recently estimated a magnetic field in spicules as $10-26$ G. They used the observed wave length from Paper I and the oscillation period of 1 min from Nikolsky \& Platova (1971).

Figure 6 shows that most expected periods are $\sim 35 \mathrm{~s}$ and $90 \mathrm{~s}$. Then the phase speed can be estimated either as $\sim 100 \mathrm{~km} \mathrm{~s}^{-1}$ or $\sim 40 \mathrm{~km} \mathrm{~s}^{-1}$. Both of them are higher than the adiabatic sound speed for a temperature of $\sim 15000 \mathrm{~K}$ at a height of $6000 \mathrm{~km}$ (Beckers 1972), which gives $\sim 20 \mathrm{~km} \mathrm{~s}^{-1}$. Therefore we argue that observed spatial periodicity in Doppler velocity with $\sim 3500 \mathrm{~km}$ (Paper I) is caused by the propagation of kink waves with a period of 30-40 s. Then the kink speed in spicules at the heights of $3800-8700 \mathrm{~km}$ from the photosphere can be estimated as

$c_{\mathrm{k}}=90-115 \mathrm{~km} \mathrm{~s}^{-1}$.

Note that the Fourier phase difference, roughly estimated for the oscillation in spicule IV (feature B), also gives a similar value for the phase speed ( $110 \mathrm{~km} \mathrm{~s}^{-1}$, see previous section).

Plasma density is much higher inside spicules than outside, therefore the kink speed is close to the Alfvén speed. Expected electron density in spicules at the height of $6000 \mathrm{~km} 8.9 \times$ $10^{10} \mathrm{~cm}^{-3}$ (Beckers 1972) gives the plasma density of $\rho_{0} \approx 1.4 \times$ $10^{-13} \mathrm{~g} \mathrm{~cm}^{-3}$. Then, using the kink speed (5), we may estimate the magnetic field strength in spicules at the height of $6000 \mathrm{~km}$ as

$B_{0}=12-15 \mathrm{G}$.

This is in good agreement with recently estimated magnetic field strengths in quiet-Sun spicules $(\sim 10 \mathrm{G})$, which was obtained by spectropolarimetric observations of solar chromosphere in He I $\lambda 10830$ (Trujillo Bueno et al. 2005).

\subsection{Some critical points}

However, there are some critical points in interpretations of observational data.

1. Fine structure of spicules: it is quite possible that spicules consist of several thinner magnetic tubes with a $100-200 \mathrm{~km}$ diameter and that this fine structure is not resolved due to the spatial resolution of observations ( $\sim 1 \mathrm{arcsec})$. Then each spicule may consist of several micro spicules. Each micro spicules may oscillate in its own way, which complicates the spectral analysis of integrated Doppler shift.

2. Line-of-sight effect of several spicules: some spicules show a multi-component structure (i.e. several spicules are located close together along a line of sight) mostly at lower heights, which may give the impression of a velocity shift (Xia et al. 2005). Therefore we chose only the spicules with well-defined single-component structure; however the lineof-sight problem cannot be completely excluded.

\section{Conclusion}

Here we analysed the old $\mathrm{H} \alpha$ spectra of the solar limb spicules taken on the coronagraph of Abastumani Astrophysical Observatory. The time series includes whole lifetimes of four different spicules at 8 different heights covering $3800-8700 \mathrm{~km}$ above the photosphere (totally 32 time series). After the DFT of the time series, we conclude:

- Doppler velocities of spicules undergo oscillations with periods of 20-110 s; 
- there are two different ranges of periods; almost half of the oscillatory periods are located in the range of 20-55 $\mathrm{s}$ and almost one third of the oscillatory periods are located in $75-110 \mathrm{~s}$;

- the most pronounced oscillation periods are $\sim 35$ and $\sim 90 \mathrm{~s}$;

- there is an interesting peak at the $178 \mathrm{~s}$ period, which is clear evidence of 3-min oscillations at the heights of $3800-8700 \mathrm{~km}$ (detection of 5-min period in our data is restricted due to the lifetimes of spicules);

- we suggest that the oscillations are caused by wave propagation in the chromosphere;

- the waves or periodic flows are likely generated in the photosphere by granular cells;

- the two different ranges of periods can be explained either by fast and slow waves or by some unknown spatial scaling of granular cells;

- observations can be used to infer plasma parameters in spicules, i.e. spicule seismology;

- estimated magnetic field strength in spicules is $12-15 \mathrm{G}$ at $\sim 6000 \mathrm{~km}$ above the photosphere.

However, some problems may arise in interpreting of observational data related to spicule fine structure or its multicomponent nature, which may lead to an impression of a velocity shift (Xia et al. 2005). Therefore future detailed observations with higher spatial resolutions (Solar Optical Telescope at Hinode spacecraft) are needed to confirm high-frequency wave propagations in the chromosphere.

Acknowledgements. The work was supported by the Georgian National Science Foundation grant GNSF/ST06/4-098. A part of the work is supported by the ISSI International Programme "Waves in the Solar Corona". We thank Prof. Kiladze for helpful comments and the referee for stimulating suggestions.

\section{References}

Ballester, J. L. 2006, Space Sci. Rev., 122, 129

Beckers, J. M. 1972, ARA\&A, 10, 73

DeForest, C. E. 2004, ApJ, 617, L89

De Pontieu, B., Erdélyi, R., \& de Wijn, A. G. 2003, ApJ, 595, L63

De Pontieu, B., Erdélyi, R., \& James, S. P. 2004, Nature, 430, 536

Edwin, P. M., \& Roberts, B. 1983, Sol. Phys., 88, 179

Hasan, S. S., \& Kalkofen, W. 1999, ApJ, 519, 899

Katsiyannis, A. C., Williams, D. R., McAteer, R. T. J., et al. 2003, A\&A, 406, 709

Khutsishvili, E. 1986, Sol. Phys., 106, 75

Kukhianidze, V., Zaqarashvili, T. V., \& Khutsishvili, E. 2005, Proceedings of the 11th European Solar Physics Meeting, The Dynamic Sun: Challenges for Theory and Observations, Leuven, Belgium, ed. D. Danesy, S. Poedts, A. De Groof, \& J. Andries, ESA SP-600, 64

Kukhianidze, V., Zaqarashvili, T. V., \& Khutsishvili, E. 2006, A\&A, 449, L35 (Paper I)

Kulidzanishvili, V. I., \& Zhugzhda, Y. D. 1983, Sol. Phys., 88, 35

Hollweg, J. V. 1981, Sol. Phys., 70, 25

Mishonov, T. M., Maneva, Y. G., \& Hristov, T. S. 2007, J. Atmosph. and SolarTerrestrial Phys., in press

Molowny-Horas, R., Oliver, R., Ballester, J. L., \& Baudin, F. 1997, Sol. Phys., 172,181

Nakariakov, V. M., \& Ofman, L. 2001, A\&A, 372, L53

Nikolsky, G. M., \& Platova, A. G. 1971, Sol. Phys., 18, 403

Roberts, B. 1979, Sol. Phys., 61, 23

Singh, K. A. P., \& Dwivedi, B. N. 2007, New Astron., 12, 479

Spruit, H. C. 1981, A\&A, 98, 155

Sterling, A. C. 2000, Sol. Phys., 2000, 196, 79

Trujillo Bueno, J., Merenda, L., Centeno, R., Collados, M., \& Landi Degl'Innocenti, E. 2005, ApJ, 619, L191

Xia, L. D., Popescu, M. D., Doyle, J. G., \& Giannikakis, J. 2005, A\&A, 438, 1115

Zaqarashvili, T. V. 2003, A\&A, 399, L15

Zaqarashvili, T. V., \& Roberts, B. 2002, Phys. Rev. E, 66, 026401

Zaqarashvili, T. V., \& Murawski, K. 2007, A\&A, 470, 353

de Wijn, A. G., Rutten, R. J., \& Tarbel, T. D. 2005, A\&A, 430, 1119

Wunnenberg, M., Kneer, F., \& Hirzberger, J. 2002, A\&A, 395, L15 